\title{
Molecular engineering of antiferromagnetic rings for quantum computation
}

\author{
F. Troiani,,${ }^{1,}$ A. Ghirri, ${ }^{1}$ M. Affronte,${ }^{1}$ S. Carretta, ${ }^{2}$ P. Santini, ${ }^{2}$ \\ G. Amoretti, ${ }^{2}$ S. Piligkos, ${ }^{3}$ G. Timco,${ }^{3}$ and R.E.P. Winpenny ${ }^{3}$ \\ ${ }^{1}$ INFM - $S^{3}$ National Research Center on nanoStructures and bioSystems at Surfaces and Dipartimento di Fisica, \\ Università di Modena e Reggio Emilia, via G. Campi 213/A, I-41100 Modena, Italy \\ ${ }^{2}$ INFM and Dipartimento di Fisica, Università di Parma, Parco Area delle Scienze, I-43100 Parma, Italy \\ ${ }^{3}$ Department of Chemistry, University of Manchester, \\ Oxford Road, Manchester M139PL, United Kingdom
}

(Dated: October 25, 2018)

\begin{abstract}
The substitution of one metal ion in a Cr-based molecular ring with dominant antiferromagnetic couplings allows to engineer its level structure and ground-state degeneracy. Here we characterize a $\mathrm{Cr}_{7} \mathrm{Ni}$ molecular ring by means of low-temperature specific-heat and torque-magnetometry measurements, thus determining the microscopic parameters of the corresponding spin Hamiltonian. The energy spectrum and the suppression of the leakage-inducing $S$-mixing render the $\mathrm{Cr}_{7} \mathrm{Ni}$ molecule a suitable candidate for the qubit implementation, as further substantiated by our quantum-gate simulations.
\end{abstract}

PACS numbers: 75.50.Xx, 03.67.-a, 75.40.Cx

Due to their relative decoupling from the environment and to the resulting robustness, electron spins in solidstate systems are currently considered among the most promising candidates for the storing and processing of quantum information (QIP) [1]. In this perspective, an increasing interest has recently been attracted by a novel class of molecular magnets, including both ferromagnetic 2] and antiferromagnetic [3] systems. In the latter case the quantum hardware is thought as a collection (e.g., a planar array) of coupled molecules, each corresponding to a different qubit. A major advantage with respect to analogous schemes based on single-spin encodings would arise from the larger dimensions of the physical subsystem, and on the resulting reduction of the spatial resolution which is required for a selective addressing of each qubit by means of local magnetic fields [1]. A detailed, system-specific investigation is however mandatory in order to verify the actual feasibility of this approach, and to suitably engineer the intra- and intercluster interactions, the coupling between the computational and the environmental degrees of freedom, as well as the gating strategy.

It is the purpose of the present Letter to argue the suitability of Cr-based antiferromagnetic molecular rings for the qubit implementation on the basis of a detailed theoretical and experimental investigation of its wavefunctions and energy levels, and of its simulated time evolution as induced by sequences of pulsed magnetic fields. Molecular rings [4] are characterized by a cyclic shape and by a dominant antiferromagnetic coupling between nearest neighbouring ions. In the absence of applied fields and for even numbers of spin centers, their energy spectrum typically consists in a singlet ground state and in characteristic rotational excitations [5]. The recently demonstrated substitution of a $\mathrm{Cr}^{3+}$ ion with a divalent transition metal [6], provides an extra spin to the otherwise fully compensated molecule: this may in turn result in the formation of a ground state doublet energetically separated from the higher energy levels, i.e. in a suitable level structure for the qubit implementation.

In the octanuclear heterometallic ring of our present concern, one of the $\mathrm{Cr}^{3+}(\mathrm{s}=3 / 2)$ ions is substituted by $\mathrm{a} \mathrm{Ni}^{2+}(\mathrm{s}=1)$ one. The cyclic molecule, with a diameter $d \sim 1 \mathrm{~nm}$, is characterized by a planar arrangement; we thus define $\theta$ to be the angle between the static magnetic field $\mathbf{B}_{0}$ and $\hat{\mathbf{z}}$, the latter being perpendicular to the ring plane. The spin Hamiltonian corresponding to the single molecular magnet reads [7]:

$$
\begin{array}{r}
\mathcal{H}=\sum_{i=1}^{8} J_{i} \mathbf{s}_{i} \cdot \mathbf{s}_{i+1}+\sum_{i=1}^{8} d_{i}\left[s_{z, i}^{2}-s_{i}\left(s_{i}+1\right) / 3\right]+ \\
\sum_{i<j=1}^{8} \mathbf{s}_{i} \cdot \mathbf{D}_{i j} \cdot \mathbf{s}_{j}+\mu_{B} \sum_{i=1}^{8} g_{i} \mathbf{B} \cdot \mathbf{s}_{i},
\end{array}
$$

where the first term accounts for the dominant isotropic exchange interaction, the second and third ones describe the anisotropic local crystal-field and the intracluster dipole-dipole interaction, respectively; isotropic $g$ factors are assumed for the last, Zeeman term. The anisotropic part of $\mathcal{H}$ does not commute with the squared total spin operator $\mathbf{S}^{2}$, and thus mixes subspaces corresponding to different values of the total spin (S-mixing). Due to its reduced symmetry, $\mathcal{H}$ can no longer be independently diagonalized within each $(2 S+1)$-dimensional block: an efficient solution scheme, based on an irreducible tensor operator formalism, has therefore been developed (see Ref. 7] and references therein) and applied to the present case.

In order to estimate the parameters entering the above spin Hamiltonian, we measure the heat capacity $C$ as a function of the temperature $(0.4<T<10 \mathrm{~K})$ and of the magnetic field $\left(0<B_{0}<7 \mathrm{~T}\right)[\underline{6}]$. The sam- 


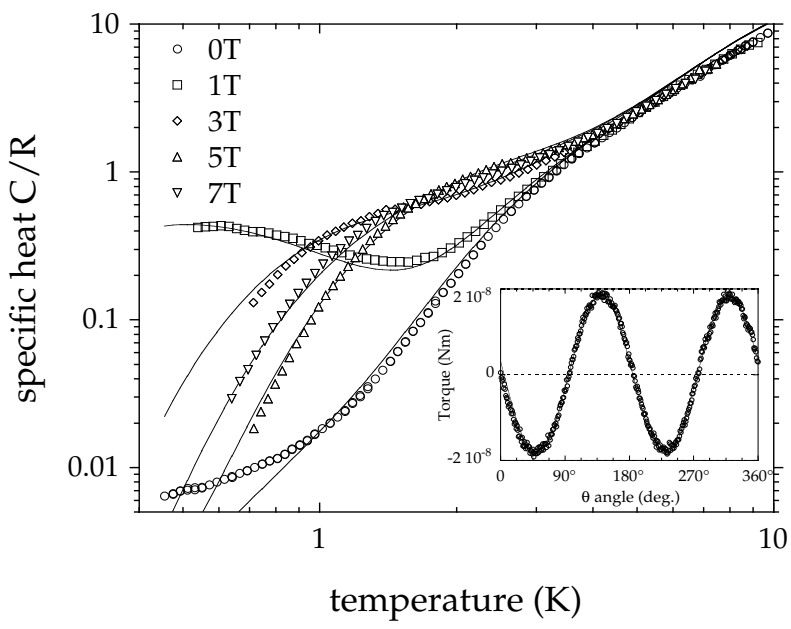

FIG. 1: Specific heat $C / R$ of a single $\mathrm{Cr}_{7} \mathrm{Ni}$ crystal as a function of the temperature $(T)$ and of the magnetic field $\left(B_{0}\right)$. We attribute the slight discrepancy between the theoretical curve and the experimental data at $B_{0}=0$ and low $T$ to an experimental artifact, probably due to the presence of defected rings within the sample. Inset: torque signal measured on a $0.7 \times 1 \times 0.5 \mathrm{~mm}^{3} \mathrm{Cr}_{7} \mathrm{Ni}$ crystal at $T=2 \mathrm{~K}$ and $B_{0}=2 \mathrm{~T}$. In our experimental set-up the rotation and torque direction is perpendicular to $\mathbf{B}_{0}$ and a positive torque induces a rotation of a negative $\theta$.

ple we investigate is a $2 \mathrm{mg}$ bulk crystal, consisting in an ensemble of independent, iso-oriented and identical rings. In fact, the synthesis procedures developed in supramolecular chemistry allow the engineering of regular arrays, as required (together with an intercluster coupling) by the long-term goal of scaling up the quantum hardware. The experimental details and the method are described in Ref. [7], while the synthesis [6] and a systematic study of the thermodynamic properties of $\left[\left\{\mathrm{Me}_{2} \mathrm{NH}_{2}\right\}\left\{\mathrm{Cr}_{7} M \mathrm{~F}_{8}\left(\mathrm{O}_{2} \mathrm{CCMe}_{3}\right)_{16}\right\}\right]$ (in short $\mathrm{Cr}_{7} \mathrm{M}$, with $\mathrm{M}=\mathrm{Fe}, \mathrm{Co}, \mathrm{Ni}, \mathrm{Mn}, \mathrm{Cd}$ ) will be reported elsewhere. In Fig. 1 we show $C\left(T ; B_{0}\right)$, normalized to the gas constant $R$, for five different values of $B_{0}$. The typical low$T$ Schottky anomaly appears upon application of the magnetic field and is shifted towards higher temperatures as $B_{0}$ increases. The overall specific heat $C\left(T ; B_{0}\right)$ arises from two distinct contributions: one is a latticerelated Debye term, $C_{\text {latt }} / R=234 r T^{3} /\left(\Theta_{D}+\lambda T^{2}\right)^{3}$, where $r=298$ is the number of atoms per molecule, $\Theta_{D}=(158 \pm 10) \mathrm{K}$ and $\lambda=(0.42 \pm 0.02) \mathrm{K}^{-1}$; the other, $C_{m}$, depends on the spin degrees of freedom and is responsible for the Schottky anomaly. The dependence of $C_{m}$ on the energy eigenvalues $\epsilon_{i}$ of $\mathcal{H}$ reads:

$$
C_{m}=R \beta^{2} \frac{\Sigma_{i} \epsilon_{i}^{2} e^{-\beta \epsilon_{i}} \Sigma_{i} e^{-\beta \epsilon_{i}}-\left(\Sigma_{i} \epsilon_{i} e^{-\beta \epsilon_{i}}\right)^{2}}{\left(\Sigma_{i} e^{-\beta \epsilon_{i}}\right)^{2}},
$$

with $\beta=1 / k_{B} T$. The best fit of the specific-heat tem- perature dependence at different values of $B_{0}$ (solid lines in Fig. (1) provides the following set of values for the microscopic physical parameters: $J_{C r} / k_{B}=(17 \pm 0.5) \mathrm{K}$, $J_{N i} / J_{C r}=0.9 \pm 0.1,\left|d_{i}\right| / k_{B}=(0.3 \pm 0.15) \mathrm{K}$ (for all the spin sites) and $g_{\mathrm{Ni}}=2.2 \pm 0.1 ; g_{\mathrm{Cr}}=1.98 \pm 0.02$ was determined from previous measurements on $\mathrm{Cr}_{8}$. The sign of the anisotropies $d_{i}$ needs to be independently determined, as we do by means of torque magnetometry measurements. In the inset of Fig. 10 we report an angular scan of the torque signal, measured at $T=2 \mathrm{~K}$ and $B_{0}=2 \mathrm{~T}$. The torque exerted, e.g., for slightly off-perpendicular magnetic-field orientations $\left(\theta \sim 90^{\circ}\right)$ tends to align the ring plane to the direction of $\mathbf{B}_{0}$. For symmetry reasons, the doublet ground state gives no contribution to the torque, and those of the first excited multiplet $(S=3 / 2$, see below) are the only excited states to be meaningfully populated at $T=2 \mathrm{~K}$ : the above behaviour therefore shows that the $\mathrm{S}=3 / 2$ manifold has an easy-plane anisotropy, which implies $d_{i}<0$. Results independently obtained by high-field torque measurements and inelastic neutron scattering (INS) confirm the above estimates of the microscopic parameters [9]. Lower-symmetry terms probably need to be included in the spin Hamiltonian in order to refine the interpretation of the INS data, but are neglected in the following since, they have little relevance to this discussion. Finally, the intracluster dipole-dipole interaction has been evaluated by means of the pointdipole approximation.

We are now able to include the microscopic parameters in $\mathcal{H}$ and accordingly draw the pattern of the low-lying energy levels $\epsilon_{i}$ as a function of $\mathbf{B}_{0}=B_{0} \hat{\mathbf{z}}$ (Fig. 2). At zero field the ground state is a degenerate doublet $\left(\epsilon_{0,1}\right)$, with a largely dominating ( $\gtrsim 99 \%)$ total-spin component $S=1 / 2$; the first excited states $\left(\epsilon_{2-5}\right)$, instead, belong to a typical rotational band, with $S \simeq 3 / 2$. Noticeably enough, these low values of the $S$-mixing allow us to consider the total spin as a good quantum number for the lowest eigenstates of the $\mathrm{Cr}_{7} \mathrm{Ni}$ molecule. As discussed in more detail in the following, two other physical quantities also play a crucial role in the perspective of a QIP implementation. The first one is the energy difference between the ground-state doublet and the higher-lying levels, i.e. $\Delta \equiv \epsilon_{2}-\epsilon_{1}$ : in fact, $\Delta$ determines to which extent the ring behaves as an effective two-level system, i.e. a meaningful population of any state but $|0\rangle$ and $|1\rangle$ can be avoided throughout the molecule manipulation. The second one is the splitting between the two $S=1 / 2$ states, $\delta \equiv \epsilon_{1}-\epsilon_{0}: \delta$ fixes the temperature the systems has to be cooled at in order for it to be initialized to its ground state. Likewise the same existence of a ground-state doublet and the suppression of the $S$-mixing, the large energy separation from the higher states in the present molecule, $\Delta(0) \simeq 13 \mathrm{~K}$, is a non-trivial result of the system engineering. Besides, the magnetic field allows a further tuning of the molecule's level structure. In particular, it increases $\delta$, 
FIG. 2: Energy levels of the $\mathrm{Cr}_{7} \mathrm{Ni}$ molecule as a function of a static magnetic field applied along the $z$-axis. At zero field the ground-state doublet is energetically separated from the higher states $(\Delta(0) \simeq 13 \mathrm{~K})$, thus representing a suitable choice for the qubit encoding.

whereas it decreases the energy difference $\Delta\left(B_{0}\right)$ between $|1\rangle \simeq|S=1 / 2, M=1 / 2\rangle$ and $|2\rangle \simeq|3 / 2,-3 / 2\rangle$. The achievement of the best trade-off between the conflicting requirements of maximizing $\delta$ and $\Delta$ therefore determines the optimal value of the field, which we identify with $B_{0}=2 \mathrm{~T}$ (see the discussion below). As a consequence, the achievable temperature required for the system initialization to the $|0\rangle$ state is $T<<\delta / k_{B} \simeq 2.4 \mathrm{~K}$, whereas $\Delta\left(B_{0}\right) \simeq \Delta(0)-2 g \mu_{B} B_{0} \simeq 9.4 \mathrm{~K}$. On the grounds of the above results, the $\mathrm{Cr}_{7} \mathrm{Ni}$ molecule can be considered as an effective $S=1 / 2$ spin cluster, and the information states $|0\rangle$ and $|1\rangle$ safely identified with its ground-state doublet. Such conclusion is unaffected by the uncertainty on $\Delta(0)$ (resulting from that on the microscopic parameters) which is roughly equal to that on $J_{C r}$ [10].

The time simulation of the quantum gates provides an important feedback for the optimization of the physical parameters. In fact, the general unitary transformation applied to the computational space is decomposed into a sequence of elementary gates, such as the $S U(2)$ rotations of the single qubit and the two-qubit CNOT [11]. We start by considering the former, which can be obtained as a combination of 3 rotations about any two orthogonal axes, e.g. $U(\alpha, \beta, \gamma)=\exp \left(-i \alpha \sigma_{2}\right) \exp \left(-i \beta \sigma_{1}\right) \exp \left(-i \gamma \sigma_{2}\right)$, being $\sigma_{1,2,3}$ the Pauli matrixes. Transitions between the $|0\rangle$ and $|1\rangle$ states, i.e. rotations about the $x$ and $y$ axes, can be induced by means of resonant, in-plane electromagnetic pulses $B_{1}(t) \cos \omega t$, where $B_{1}(t) \ll B_{0}$ represents the slowly-varying envelope. In the case of an effective two-level system, however, the transverse magnetic field also couples the $|0\rangle$ and $|1\rangle$ states to the higher-lying ones, thus inducing a population loss (leakage) during gating, quantified by $L=1-\left[|\langle 0 \mid \psi(t)\rangle|^{2}+|\langle 1 \mid \psi(t)\rangle|^{2}\right]$. More specifically, the occurrence of such unwanted transitions is due to the $S$-mixing and to possible intracluster inhomogeneities in the magnetic fields (or, equivalently, in the ion $g$ factors): both result in non-vanishing matrix elements $\left\langle 0,1\left|\mathcal{H}_{o p}\right| i \geq 2\right\rangle$, with $\mathcal{H}_{o p}=\mu_{B} \sum_{i=1}^{8} g_{i} \mathbf{B}_{1} \cdot \mathbf{s}_{i}$. Together with a molecular engineering aimed at the suppression of the $S$-mixing, the minimization of $L$ can be achieved by the use of "soft" enough pulses, i.e. by keeping the pulse spectral dispersion $\Delta \omega<(\Delta-\delta) / \hbar$. Being $\Delta \omega \sim 1 / \tau_{g}$, such inequality gives a lower bound to the the ratio $\tau_{g} / \tau_{d}$ that can in principle be achieved $\left(\tau_{g, d}\right.$ are the gating and decoherence time, respectively).

Here, by means of a numerical integration of the Schrödinger equation, we calculate the time dependence of $\left|c_{0,1}\right|^{2}=|\langle 0,1 \mid \psi(t)\rangle|^{2}$ and of $L$ corresponding to a $\pi$ rotation about the $x$ axis, for $|\psi(0)\rangle=|0\rangle$ and $B_{0}=2 \mathrm{~T}$ (see Fig. 33); the pulsed field is assumed to have a gaussian profile $B_{1}(t)=A \exp \left[-\left(t-t_{0}\right)^{2} /\left(2 \sigma^{2}\right)\right]$, with $A=0.1 \mathrm{~T}$ and $\sigma=150 \mathrm{ps}$. To a very large extent the leakage involves the first excited multiplet, and its value remains lower than $10^{-5}$ throughout the time evolution: the lower limit of $\tau_{g}$ imposed by the presence of upper levels is thus by far larger than the one arising, e.g., from the present technological limitations in the pulse generation. Further simulations, performed with more reasonable values of the magnetic field $(A=0.01 \mathrm{~T})$ [12], give proportional increases of the pulse duration, witht a further suppression of $L$. We have also investigated the gate robustness with respect to possible spatial inhomogeneities of $\mathbf{B}_{1}$, generally leading to a larger values of $L$ : even in the worst limiting case of a $\mathbf{B}_{1}$ which is nonzero only at the Ni-ion site, $L$ remains below the threshold of $10^{-4}$ ( $\left.A=0.15 \mathrm{~T}, \tau_{g} \sim 2 \sigma \sim 300 \mathrm{ps}\right)$. A larger value of the static field $B_{0}$ would polarize nuclear spins, thus reducing the decoherence due to hyperfine field fluctuations (see below). For $B_{0}>5.6 T, \epsilon_{1}>\epsilon_{2}$ and $|1\rangle$ can relax into $|2\rangle$ by emitting a phonon (the decay to $|0\rangle$ is practically forbidden, owing to the approximate Kramers-doublet nature of the computational basis). These processes, however, are expected to be completely negligible with respect to other sources of decoherence, due to the small value of $\left(\epsilon_{1}-\epsilon_{2}\right) / k_{B} \Theta_{D}$. An increase of $L$ might arise from the breakdown of the condition that $\Delta>\Delta \omega$. In order to estimate this effect, we have considered a static field $B_{0}=5.61 \mathrm{~T}$, which implies $\Delta \simeq 0$ (Fig. Bb): $L$ is seen to reach $\sim 3 \%$, but even small deviations of $B_{0}$ from this critical values is enough to suppress $L$.

The experimental demonstration of the intercluster coupling required for the implementation of the two-qubit gates is beyond the scope of the present work. In the following we discuss the present scenario and possible strategies to be pursued in order to achieve the required conditional dynamics. In particular, links between $\mathrm{Cr}_{7} \mathrm{Ni}$ rings formed by delocalized aromatic molecules have already been synthesized [13], and intercluster couplings have been demonstrated in similar systems 14]. The simplest case occurs when the coupling between the rings results from the interaction of the $m$-th spin in molecule $I$ with the $n$-th of $I I$. If we apply the first-order perturbation theory and restrict ourselves to the product space $\{|0\rangle,|1\rangle\}_{I} \otimes\{|0\rangle,|1\rangle\}_{I I}$, a Heisenberg interaction $\mathcal{H}_{I, I I}=J^{*} \mathbf{s}_{m}^{I} \cdot \mathbf{s}_{n}^{I I}$ can be rephrased as follows in terms of the cluster total spins:

$$
\mathcal{H}_{I, I I}=J_{\text {eff }} \mathbf{S}_{I} \cdot \mathbf{S}_{I I},
$$

where

$$
J_{\text {eff }}=2 / 3 J^{*}\left\langle 1 / 2\left\|s_{m}\right\| 1 / 2\right\rangle\left\langle 1 / 2\left\|s_{n}\right\| 1 / 2\right\rangle
$$

(analogous equations apply to the case of an Ising interaction). In the present molecules the single-cluster 
FIG. 3: Simulated time evolution of the molecular magnet, initially prepared in $|0\rangle$, under the effect of a transverse magnetic field with a gaussian temporal profile. The black, red and blue lines correspond to $\left|c_{0}\right|^{2},\left|c_{1}\right|^{2}$ and $L$ (multiplied by a factor $10^{5}$ in (a)), respectively; the static field is (a) $B_{0}=2 \mathrm{~T}$ and (b) $B_{0}=5.61 \mathrm{~T}$, whereas $A=0.1 \mathrm{~T}$ in both cases.

reduced matrix elements $\left\langle 1 / 2\left\|s_{m, n}\right\| 1 / 2\right\rangle$ has a modulus of the order of unity and alternating positive-negative signs as a function of $n$ and $m$, whereas it is always negative for the $\mathrm{Ni}$ ion. If the interchain couplings are more than one, $J_{\text {eff }}$ is given by the sum of the single contributions. Therefore, $J_{\text {eff }}$ has the same order of magnitude of the coupling $J^{*}$ between single spins, whereas its site dependence provides additional flexibility to the implementation scheme.

Within the present scenario the inter-cluster couplings, though engineerable during the growth process, are untuneable thereafter. This shortcoming is common to other implementations, and doesn't in principle prevent from performing the single- and two-qubit quantum gates. In fact, depending on the form and magnitude of the interchain coupling, different approaches can be adopted. Weak and Ising-like interactions allow the use of the so-called "refocusing techniques", widely developed within the NMR community [15]. Off-diagonal (e.g., Heisenberg) intercluster interactions could instead require either multi-ring encodings of the qubit, resulting in a symmetry-induced cancellation of the logical coupling between the encoded qubits [16], or the use of interqubit molecules acting as tuneable barriers 17].

We finally discuss the decoherence of the cluster-spin degrees of freedom, which is expected to mainly arise from the hyperfine coupling with the nuclear spins. A first estimate can be obtained by considering the dipolar interaction of one $\mathrm{Cr}$ ion $(s=3 / 2)$ with the neighboring $\mathrm{F}$ nucleus (natural abundance $\sim 100 \%$ of the $I=1 / 2$ isotope). Being $g_{F}=+5.2577$ and the distance of each of the eight $\mathrm{F}$ atoms from the nearest $\mathrm{Cr}$ ion $d=0.191 \mathrm{~nm}$, the interaction energy corresponds to $E_{\text {hyp }} / k_{B}=0.38 \mathrm{mK}$. For an octanuclear ring this would give $\tau_{d} \simeq \hbar / 8 E_{\text {hyp }} \sim 2.5 \mathrm{~ns}$, that can be considered as a lower bond for $\tau_{d}$. Similar $\tau_{d}$ values have been estimated for other molecular magnets 14, 18]. Direct measurements of the electron-spin resonance linewidth on a $\mathrm{Cr}_{7} \mathrm{Ni}$ crystal (which also includes the effects of possible inhomogeneities, the so-called "dephasing") provides $\tau_{d}$ values one order of magnitude larger than the above one [19]. Substantial enhancements of $\tau_{d}$ will result from the suppression of the hyperfine field fluctuations (mK temperatures and few-T static fields) and from the substituting of the $\mathrm{F}$ ions with $\mathrm{OH}$ groups in the $\mathrm{Cr}_{7} \mathrm{Ni}$ compound, which we are already working at. Besides, the static field induces a large mismatch between the en- ergy gaps of the nuclear and electronic spins, thus rendering highly inefficient the relaxation processes of the latter. A second potential source of decoherence, namely the ring-ring dipolar coupling, is characterized by a lower energy scale, $E_{d i p} / k_{B} \simeq\left(g \mu_{B} S\right)^{2} / V \sim 0.1 \mathrm{mK}(S=1 / 2$ and $V=6.346 \mathrm{~nm}^{3}$ ), futher reduceable in diluted $\mathrm{Cr}_{7} \mathrm{Ni}$ molecular systems.

In conclusion, the energy spectrum of the investigated $\mathrm{Cr}_{7} \mathrm{Ni}$ molecule fully justifies its description in terms of an effective two-level system; besides, the symmetries of the ground-state doublet ( $S$-mixing below $1 \%)$ suppress the coupling to the higher levels as induced by the transverse magnetic fields which are required for the quantum-gate implementation. In fact, our simulations of the single-qubit gates provide negligible values for the leakage $\left(L \lesssim 10^{-4}\right)$ even for gating times of the order of $10^{2}$ ps, i.e. well below the tens of ns estimated for the spin decoherence times. While further work is needed for the engineering of the intercluster coupling, these results strongly support the suitability of the $\mathrm{Cr}_{7} \mathrm{Ni}$ rings for the qubit encoding and manipulation.

* Corresponding author: troiani.filippo@unimore.it

[1] D. Awschalom, N. Samarth, D. Loss, Semiconductor Spintronics and Quantum Computation (Springer, Berlin, 2002).

[2] M.N. Leuenberg, D. Loss, Nature 410, 789 (2001).

[3] F. Meier, J. Levy, D. Loss, Phys. Rev. Lett. 90, 047901 (2003); Phys. Rev. B 68, 134417 (2003).

[4] K.L. Taft et al., J.Am. Chem. Soc. 116, 823, (1994);

[5] J. Schnack and M. Luban, Phys. Rev. B 63, 014418 (2000); O. Waldmann et al., Phys. Rev. Lett. 91, 237202 (2003).

[6] F.K. Larsen et al., Angew. Chem. Int. Ed. 42, 101 (2003).

[7] S. Carretta et al., Phys. Rev. B 67, 094405 (2003); M. Affronte et al., Eur. Phys. J. B 15, 633 (2000); Appl. Phys. Lett. 84, 3468 (2004).

[8] S. Carretta, A. Ghirri, M. Affronte, G. Amoretti, S. Piligkos, R.E.P. Winpenny, unpublished.

[9] S. Carretta et al., cond-mat/0412628 R. Caciuffo et al., cond-mat/0412169

[10] The lowest eigenstates of the AF ring can be approximately described by means of the sublattice spin operators (where all the odd spins are assumed to be parallel to each other, and so the even ones): $\mathbf{S}_{A}=$ $\sum_{i=1}^{4} \mathbf{s}_{2 i-1}, \mathbf{S}_{B}=\sum_{i=1}^{4} \mathbf{s}_{2 i}$. The dominant term in $\mathcal{H}$ is thus $J_{i} / 2 \mathbf{S}_{A} \cdot \mathbf{S}_{B}$, which gives a splitting of $3 / 2 J_{i}$ between $S=3 / 2$ and $S=1 / 2$.

[11] M.A. Nielsen and I.L. Chuang, Quantum Computation and Quantum Information, (Cambridge University Press, Cambridge, 2000).

[12] D.A. Lidar and J. H. Thywissen, J. Appl. Phys. 96, 754 (2004).

[13] G. Timco, R.E.P. Winpenny to be published.

[14] S. Hill et al., Science 302, 1015 (2003).

[15] See, e.g., J.A. Jones and E. Knill, J. Magn. Reson. 141, 322 (1999). 
[16] X. Zhou et al., Phys. Rev. Lett. 89, 197903 (2002).

[17] S.C. Benjamin and S. Bose, Phys. Rev. Lett. 90, 247901 (2003).
[18] E. del Barco et al., cond-mat/0405331

[19] W. Wernsdofer, private communication. 


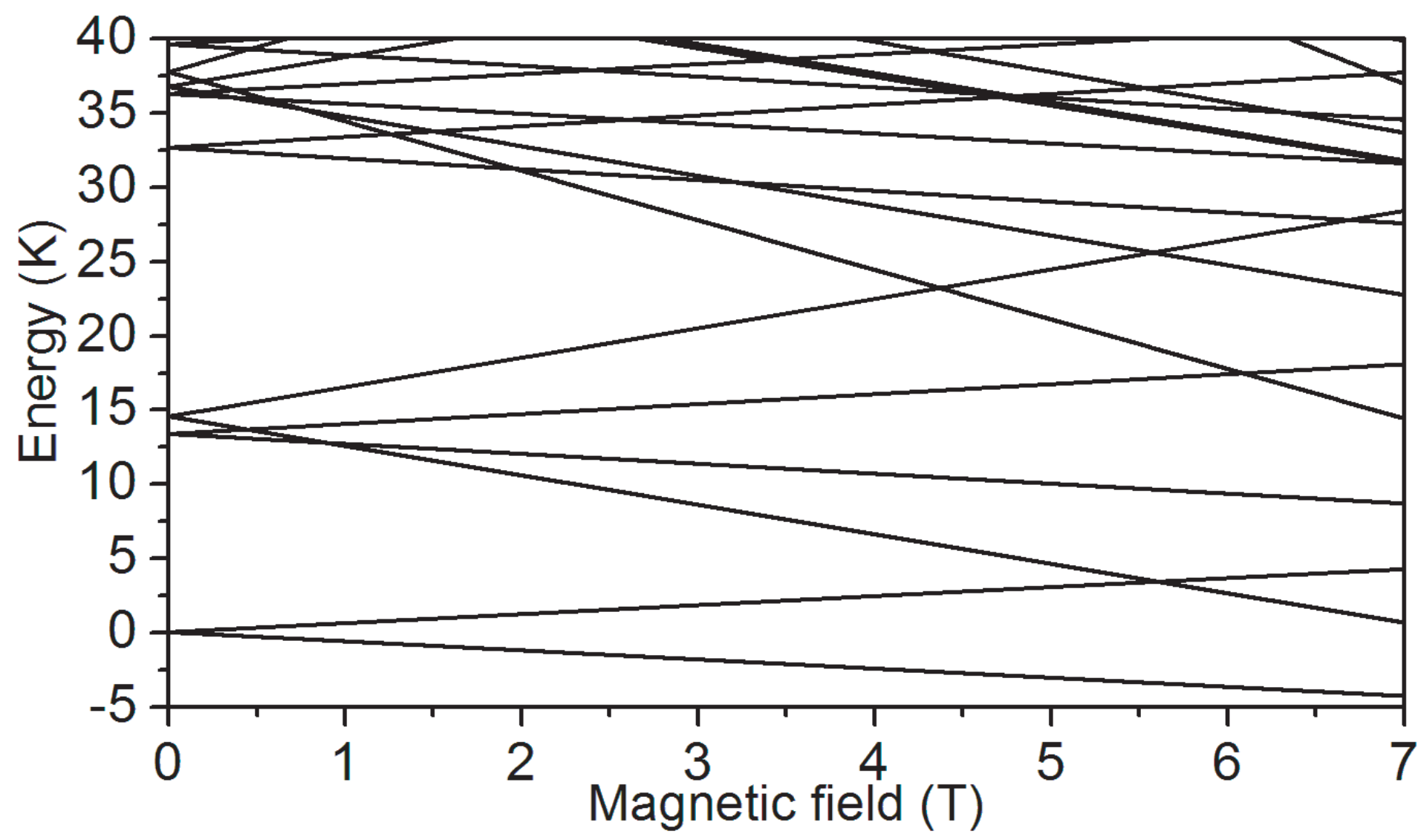




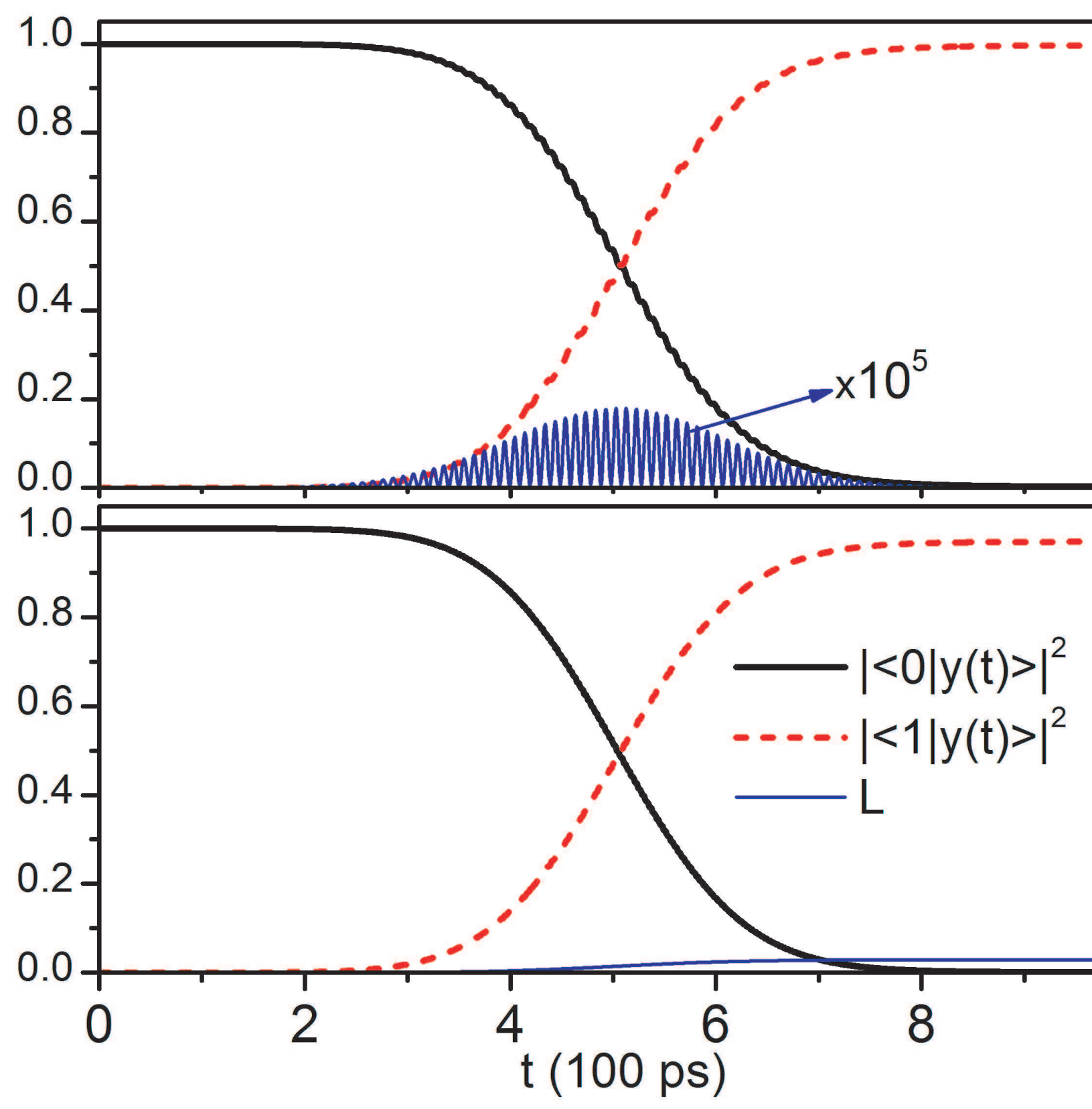

